\title{
Un estudio local sobre los factores, estrategias y canales de difusión y promoción cultural
}

\author{
A local study about the factors, strategies and channels of cultural \\ dissemination and promotion
}

Jesús Eduardo Oliva Abarca

Universidad Autónoma de Nuevo León (México)

jesus.olivaabr@uanl.edu.mx

\section{Resumen}

En este artículo se presentan los resultados de una investigación cuyo objeto lo constituyen los aspectos y las circunstancias implicados en la difusión y promoción de bienes y servicios culturales. Dicho estudio se circunscribe a Nuevo León, estado situado al norte de México; la investigación fue realizada con un enfoque cualitativo y se centró en los sub-sectores culturales de las artes visuales, la producción audiovisual y el diseño gráfico. La estrategia de recolección de datos utilizada fue la entrevista semiestructurada, que se aplicó a un total de treinta agentes culturales locales. Los resultados obtenidos se clasificaron en tres temas generales: el de los factores, el de las estrategias y el de los canales que los y las agentes culturales consideran como los más relevantes para difundir y promover sus actividades. Tras el análisis de la 
información recabada, se llegó a la conclusión de que los creadores y las creadoras culturales recurren con más frecuencia a los canales digitales, dado que son más accesibles que los canales tradicionales de difusión y promoción cultural.

Palabras clave: artes; agente cultural; canales; difusión; promoción.

\section{Abstract}

This paper presents the results of a research about the aspects and circumstances that are implied in the dissemination and promotion of cultural goods and services. This study is limited to the Mexican state of Nuevo León. The research was conducted through a qualitative approach and centered on the cultural sub-sectors of visual arts, audiovisual production, and graphic design. For collecting data, the semistructured interview was applied to thirty local cultural agents. The research results were classified into three main themes: the factors, the strategies, and the channels that cultural agents consider as the most relevant for promoting their activities. After analyzing this information, it was concluded that cultural creators use digital channels more frequently because they are more accessible than traditional channels for cultural dissemination.

Keywords: arts; channels; cultural agent; dissemination; promotion.

Son tres las etapas o fases sucesivas que caracterizan al mundo de las artes y de la cultura: primeramente, un proceso de producción, durante el cual los y las agentes generan bienes y servicios culturales diversos; segundo, el proceso de distribución, fase en la que las manifestaciones culturales son difundidas y promovidas a través de diferentes canales y con el apoyo de diversos intermediarios; finalmente, el proceso de recepción, en el cual los destinatarios reciben e interpretan los contenidos culturales según los códigos estéticos y simbólicos que tienen a su disposición (Becker, 2008). En el caso concreto de la fase de distribución, este proceso se desarrolla en tres contextos: el directo, en el que están involucrados agentes y organismos propiamente culturales; el indirecto, en el que participan individuos y grupos pertenecientes a otros ámbitos del quehacer humano, tales como administradores, contadores, abogados, etcétera; y, por último, el contexto social, que se refiere a cuestiones tales como la situación económica, laboral, el avance tecnológico, la calidad de vida, etcétera, situaciones todas que afectan la distribución cultural (Maanen, 2009: 243-244) 
Las Nuevas Tecnologías de la Información y de la Comunicación han introducido cambios significativos no sólo en la forma en la que se producen y distribuyen los mensajes de manera remota, sino también en las interacciones sociales y en todos los campos de actuación humana (Castells, 2001), incluido el ámbito cultural, cuya estructura organizativa comienza a adaptarse a nuevos modelos de producción, distribución y recepción (Goriunova, 2012). Una de las transformaciones más destacable es la de la glocalidad (Castells, 2001: 268), fenómeno propiciado principalmente por el internet y que consiste en la conexión e interacción simultánea de lo local y lo global (p. 217). En el caso particular de los bienes y servicios culturales, tales como obras de arte, eventos y exposiciones, etcétera, internet ha permitido una difusión y promoción a gran escala de las manifestaciones culturales más diversas, garantizando a las audiencias más diversas un acceso más democrático a la cultura (Rama, 1999).

La presente investigación tiene por objeto el análisis de los factores, circunstancias y aspectos que intervienen en la fase de distribución cultural; para abordar tal fenómeno es necesario acotar el estudio a un espacio geográfico, cultural y político claramente demarcado, además de especificar los sectores o sub-sectores culturales (PNUD, 2014), que serán examinados. Por tales motivos, este estudio se circunscribe a Nuevo León, estado ubicado al norte de México; las áreas o sub-sectores culturales investigados son los de las artes visuales, la producción audiovisual y el diseño gráfico. Cabe señalar que, dado que la distribución cultural implica tres contextos diferentes, según lo señalado por Maanen (2009), la presente investigación se limita al estudio del contexto directo, cuyo funcionamiento y operación está determinado por las acciones y actividades de los mismos y las mismas agentes culturales. La finalidad de este análisis es obtener la suficiente información de los y las participantes que permita esbozar una descripción de las formas y modos mediante las que las y los agentes culturales difunden y promocionan sus actividades en el estado.

Es necesario comentar, así sea de manera sucinta, las características generales del lugar en el que se desarrolla este estudio. Nuevo León es uno de los estados con mayor grado de industrialización en México, lo que ha propiciado dos situaciones: la primera, que las profesiones más valoradas sean aquellas relacionadas con las ingenierías, y la segunda, que las prácticas relacionadas con la cultura sean consideradas como actividades poco redituables (Amores, 2007). La mayoría de las iniciativas culturales en el estado han sido resultado de la inversión de empresarios y empresarias locales, así como de artistas, promotores y promotoras y educadoras y educadores interesados en el desarrollo cultural (Rubio Elosúa, 2000). Pueden listarse entre estas organizaciones privadas galerías como Drexel Proyectos y Arte A. C., esta última se ha consolidado como una institución educativa en la localidad (Amores, 2007).

Dos casos que conviene mencionar son el Museo de Arte Contemporáneo (MARCO) y el Parque Fundidora, ambos resultado de la colaboración entre instituciones públicas y empresas privadas (Nivón Bolán y Villalobos Audifred, 2006) y que son considerados como espacios 
culturales emblemáticos de Nuevo León. Por otra parte, el Consejo para la Cultura y las Artes de Nuevo León es la institución pública encargada de procurar el desarrollo de todas las disciplinas artísticas y de las diversas manifestaciones culturales en el estado. Si bien existe un creciente y renovado interés por las artes y la cultura en Nuevo León, la brecha entre la capacidad de producir bienes y servicios culturales y la posibilidad de distribuirlos de manera constante y amplia es evidente (Rubio Elosúa, 2000), principalmente por el hecho de que los recursos y apoyos para la creación son limitados, además de que, a partir de 2013 a la fecha, han sido constantes los recortes al presupuesto destinado a la cultura. Esta situación conduce a la búsqueda, por parte de los y las agentes culturales, de alternativas plausibles, y sobre todo asequibles, de difusión y promoción para sus actividades.

\section{Metodología}

El enfoque metodológico pertinente para esta investigación es el cualitativo, dado que son las percepciones, las opiniones y las experiencias de los individuos involucrados en una situación social en específico las que determinan el objeto del presente estudio (Álvarez-Gayou Jurgenson, 2012). La investigación cualitativa se centra en los sujetos, y en sus acciones y comportamientos en un escenario particular para intentar explicar fenómenos sociales; en otras palabras, el enfoque cualitativo "pretende conceptuar sobre la realidad, con base en la información obtenida de la población o las personas estudiadas" (Bernal, 2010: 60). El propósito de este estudio es examinar las circunstancias y aspectos que intervienen en la promoción y difusión cultural en Nuevo León, México, específicamente en los sub-sectores de las artes visuales, la producción audiovisual y el diseño gráfico. La pregunta de investigación que sive de punto de partida para este estudio es la siguiente: ¿qué factores, estrategias y canales emplean los y las agentes culturales neoleoneses para difundir y promover sus actividades?

Para responder a tal interrogante, se requirió diseñar la investigación en tres fases: la primera etapa consistente en la decisión muestral, la segunda es la de elección de las técnicas de recolección de datos; y la tercera y última es la referente al análisis de la información recabada. En lo que respecta a la primera fase, los y las participantes fueron seleccionados con base a dos criterios de pertinencia para la investigación, el de heterogeneidad (Valles, 1999) y el de involucramiento en la situación a analizar. El primer criterio contempla la diversidad de los casos a estudiar, lo que significa que los y las participantes reúnen características sociodemográficas diferentes, tales como la edad, los estudios y el sub-sector cultural en que se desempeñan. El criterio de involucramiento toma en consideración la experiencia profesional en años de cada participante, así como si han laborado en alguna organización cultural pública, 
privada o educativa, o si son agentes culturales independientes, sea como autoempleados, emprendedores o freelancers. En las tablas 1 y 2 se muestran los perfiles de las entrevistadas y los entrevistados según los criterios de selección previamente mencionados.

\begin{tabular}{|c|c|c|c|c|c|c|c|}
\hline \multirow{3}{*}{$\begin{array}{c}\text { Total de } \\
\text { participantes: } \\
30\end{array}$} & \multicolumn{2}{|c|}{ Edad } & \multicolumn{2}{|c|}{ Grado de estudios } & \multicolumn{3}{|c|}{ Sub-sector cultural } \\
\hline & $20-35$ & +35 & Licenciatura & Posgrado & $\begin{array}{c}\text { Artes } \\
\text { visuales }\end{array}$ & $\begin{array}{l}\text { Producción } \\
\text { audiovisual }\end{array}$ & $\begin{array}{l}\text { Diseño } \\
\text { gráfico }\end{array}$ \\
\hline & 21 & 9 & 24 & 6 & 10 & 10 & 10 \\
\hline
\end{tabular}

Tabla 1. Criterio de heterogeneidad. Fuente: elaboración propia.

\begin{tabular}{c|c|c|c|c|c|c|}
\hline $\begin{array}{c}\text { Total de } \\
\text { participantes: }\end{array}$ & \multicolumn{2}{|c|}{ Experiencia (en años) } & \multicolumn{3}{|c|}{ Adscripción } \\
\hline 30 & $1-5$ & $5-10$ & +10 & $\begin{array}{c}\text { Institucional } \\
\text { pública }\end{array}$ & $\begin{array}{c}\text { Institucional } \\
\text { privada }\end{array}$ & Independiente \\
& 4 & 17 & 9 & 9 & 14 & 7 \\
\hline
\end{tabular}

Tabla 2. Criterio de involucramiento. Fuente: elaboración propia.

Durante la fase de elección de técnicas de recolección de datos, la estrategia elegida fue la entrevista semiestructurada, que posibilita que la 0 el participante exprese de manera espontánea sus opiniones, experiencias y valoraciones respecto al fenómeno investigado (Álvarez-Gayou Jurgenson, 2012). La entrevista fue diseñada empleando cinco preguntas abiertas referentes a las dificultades, recursos, herramientas y circunstancias que condicionan la difusión y promoción cultural en Nuevo León. Para la etapa de análisis de información, se establecieron códigos a priori a partir de los cuales se examinaron los datos provistos por las y los participantes y, posteriormente, se generaron sub-códigos basados en las respuestas brindadas por los entrevistados y las entrevistadas, y cuya finalidad fue la de brindar una descripción objetiva del fenómeno estudiado. Los resultados obtenidos de esta última fase fueron clasificados en tres temas generales que a continuación se detallan.

\section{Resultados}

El objetivo de este estudio es examinar qué factores intervienen en la promoción y difusión de las actividades de agentes culturales involucrados en los sub-sectores de las artes visuales, la 
producción audiovisual y el diseño gráfico. El enfoque metodológico pertinente para ello es el cualitativo, y la técnica de recolección de información empleada fue la entrevista semiestructurada, que se aplicó de manera individual a un total de treinta agentes culturales, seleccionados de acuerdo a dos criterios de conveniencia para la investigación: heterogeneidad e involucramiento en la situación analizada. Como se indicó más detalladamente en el apartado anterior, las y los agentes culturales escogidos conforme a tales pautas poseen perfiles sociodemográficos variados, además de que participan de manera activa y constante en los ámbitos de la producción artístico-visual, audiovisual o de la comunicación gráfica ya sea como creadores, promotores, gestores o cumpliendo todas estas funciones de manera simultánea (véase Tabla 1 y 2).

La información recabada a través de las respuestas provistas por los entrevistados se analizó con base a categorías generales establecidas a priori, que a su vez posibilitaron la generación de otros códigos o sub-categorías particulares sustentadas en la realidad del fenómeno estudiado. Los resultados de la presente investigación se exponen organizados de la siguiente manera: a. factores que garantizan difusión y promoción, esto es, las circunstancias que influyen en el éxito promocional de bienes y servicios culturales; b. estrategias de inserción y/o captación, tema que se refiere a los procedimientos que las y los agentes culturales consideran como los más adecuados para lograr una mejor inserción en el ámbito cultural o una mayor captación de público; y c. canales de difusión y promoción, tema en el que se aborda los tipos de medios de los que se sirven los y las agentes culturales para promover sus obras o los servicios que ofrecen, y cuya elección se basa tanto en los factores y estrategias de difusión, así como en los recursos y posibilidades de los que dispone cada agente cultural.

\section{Factores de difusión y promoción cultural}

Este tema se refiere a las circunstancias que intervienen en la etapa de difusión o promoción de un bien o servicio de índole cultural. De acuerdo con la información proporcionada por los y las agentes culturales que participaron en esta investigación, los factores principales que garantizan una difusión exitosa de las actividades que se desarrollan son, primeramente, los espacios institucionales en los que se exhibe la obra o su proceso, o donde se brinda el servicio ofrecido; y, en segundo lugar, las elaboraciones ideológicas (García Canclini, 2014) realizadas por otros artistas, críticos, entusiastas, periodistas y demás intermediarios pertenecientes al campo cultural. En esta segunda sub-categoría se incluye la opinión de los destinatarios y destinatarias de los bienes y/o servicios culturales, esto es, de la audiencia y de la clientela. 


\section{Espacios institucionales}

Un espacio institucional pudiera definirse como un lugar o entorno, sea físico o simbólico, que opera bajo un régimen de hábitos, comportamientos y actitudes articulados bajo una ideología común (Alexander, 2000; Williams, 1981; Certeau, 1999). Desde esta óptica, un museo, un festival artístico o cinematográfico, una galería o una empresa, pueden ser considerados como espacios institucionales, dado que operan según un esquema organizado de interacciones sociales específicas; además, las prácticas y operaciones llevadas a cabo dentro de estos ámbitos son validadas cuando son ejecutadas conforme a los marcos de actuación establecidos (Goffman, 1974). En el caso del objeto de este estudio, que son los canales y medios de promoción y difusión cultural, las actividades que los y las agentes culturales realizan con el fin de promocionar su trabajo dependen de que logren establecer relaciones específicas acordes a las expectativas de actuación de los espacios institucionales en los que se han introducido.

De acuerdo a los y las participantes, existen tres tipos de espacios institucionales que garantizan que la producción del o de la agente cultural tenga una adecuada difusión son el museo, la galería y la universidad. De los y las treinta participantes, los y las diez que se desempeñan en el área de las artes visuales aseguraron que la visibilidad de sus obras o el éxito de su labor como gestores o promotores depende del lugar en que se exhiban sus trabajos o de los vínculos que puedan establecer con instituciones y organizaciones culturales y/o educativas públicas o privadas. Los y las diez participantes que laboran en el ámbito de la producción audiovisual igualmente mencionaron que frecuentemente buscan que sus proyectos audiovisuales aparezcan en muestras o festivales organizados por universidades, por empresas o por consejerías culturales públicas. En el caso de los diez diseñadores y diseñadoras gráficas participantes en esta investigación, no es muy habitual que busquen promover su trabajo a través de los espacios institucionales listados aquí; más bien difunden sus servicios directamente a clientes potenciales, ya sea mediante recomendaciones personales o redes sociales.

\section{Elaboraciones ideológicas de segundo orden}

La comunicación de cualquier obra, hallazgo o invento está determinada por diferentes circunstancias e intermediarios cuyas acciones influyen en la percepción de los destinatarios. Los modos que dichos intermediarios eligen para difundir tal o cual mensaje usualmente comportan algún tipo de intencionalidad tácita o explícita (Thompson, 1998), lo que significa que la transmisión de tal o cual mensaje implica una ideología específica, es decir, un conjunto estructurado de ideas, intereses y actitudes (Faber, 2009). De esta manera, todo sistema de comunicación puede considerarse a su vez como un sistema de elaboraciones ideológicas. Y, 
según esta perspectiva, el arte, la ciencia, la política, en suma, toda actividad humana resulta de o conlleva una elaboración ideológica que puede denominarse de primer orden, en tanto representa de manera directa uno o varios aspectos particulares de la realidad, al tiempo que son objeto de elaboraciones que se refieren no a la realidad en sí, sino a su representación a través de elaboraciones primarias. Comentarios, reseñas, ensayos, notas, artículos y demás expresiones cuyo objeto es comunicar hallazgos, invenciones, teorías u obras, pueden ser definidas como elaboraciones ideológicas de segundo orden.

Las obras artístico-visuales y las producciones audiovisuales son a menudo objeto de estas elaboraciones ideológicas, cuya utilidad radica, como acertadamente señala García Canclini (2014) en que permiten "investigar el proceso de comunicación estética, cómo se transforman los significados propios de la obra en su circulación social, por la intervención de los difusores" (p. 94). De acuerdo a las respuestas brindadas por las y los artistas visuales, indicaron que, en efecto, la crítica especializada, las reseñas de exposiciones y las notas periodísticas conllevan a una mejor difusión de sus actividades artísticas; no obstante, agregaron, la crítica, la reseña y los comentarios sobre arte en Nuevo León, realizados por otras y otros artistas y escritores y escritoras, se distribuye generalmente a través de revistas universitarias o independientes con poca cobertura, por lo que es poco probable que audiencias no involucradas al ámbito cultural o universitario tengan acceso a estas publicaciones. En lo que respecta al periodismo, informaron que existe poco o nulo interés por parte de los boletines periodísticos por abordar eventos culturales.

En el ámbito de la producción audiovisual, es aún menor la cobertura comunicativa; salvo noticias en medios impresos universitarios y algún recordatorio en los periódicos locales acerca de producciones estudiantiles o independientes que aparecen en la cartelera de algunos cines, los y las participantes declararon que la creación de cortometrajes, mediometrajes y largometrajes en Nuevo León tiene una mínima difusión por parte de agentes que no están involucrados directamente en el sub-sector audiovisual. Por su parte, los y las participantes que se desempeñan en el área del diseño gráfico comentaron que sus actividades no suelen ser objeto de reseñas o crítica especializada, sino más bien de comentarios favorables o no favorables de clientes o usuarios de sus servicios, que conllevan a ser recomendados o a ser evitados para futuros proyectos o contrataciones.

\section{Estrategias de inserción y/o captación de audiencias}

Todo producto o servicio, incluidos los culturales, está destinado a ser adquirido, utilizado o consumido por una determinada audiencia. Para lograr dicha finalidad, el o la artista o creador y/o creadora cultural $o$, en su defecto, el promotor o promotora cultural, ha de planificar ciertas estrategias por medio de las cuales el producto o servicio se inserta en un mercado específico, 
o bien, ya introducido en un mercado, se intenta que capte una mayor cantidad de consumidores o usuarios (Colbert y Cuadrado, 2015). Con base a las respuestas provistas por las y los participantes de esta investigación, se identificaron tres estrategias principales que, en opinión de las entrevistadas y entrevistados, son las más pertinentes para una mejor promoción de sus actividades en sus respectivos sub-sectores culturales. Dichas estrategias consisten, primero, en la de la personalización del producto o servicio; segundo, en la colaboración y sinergia entre agentes culturales; y tercero, en la segmentación de mercados y públicos.

\section{Personalización de producto o servicio cultural}

La personalización es un concepto opuesto al de la masificación, y alude al hecho de adaptar el producto o el servicio a las preferencias y necesidades del usuario o consumidor. Esta estrategia es usual en las industrias culturales y creativas contemporáneas, que se apoyan enormemente en la innovación, la investigación y la tecnología, y que atienden a mercados plurales y multiculturales (Howkins, 2013; PNUD, 2014). Los y las participantes que laboran en el sub-sector de las artes visuales comentaron que en algunas ocasiones recurren a esta estrategia con el propósito de captar un mayor número de posibles compradores de sus obras, principalmente cuando realizan algún trabajo por encargo; no obstante, añaden, su producción artística está determinada principalmente por sus respectivas visiones estéticas, antes que por la preferencia del espectador. En lo que respecta al ámbito audiovisual, las y los participantes señalaron que, dados los recursos y tiempo que se requieren invertir en una obra audiovisual, es posible llevar a cabo dicha estrategia sólo para audiencias muy selectas. En lo que respecta a las diseñadoras y diseñadores gráficos, afirmaron que sus actividades profesionales se rigen precisamente por la personalización de sus productos y servicios.

\section{Colaboración y sinergia}

El trabajo colaborativo no es propiamente una estrategia de promoción y difusión cultural, sino más bien una necesidad intrínseca al desarrollo del arte como una forma de crear, o recuperar, un vínculo más genuino, una interacción más dinámica entre la obra, la y el artista y los destinatarios y destinatarias (Bourriaud, 2008); para ello, se requería abandonar el mito de la soledad artística, dado que la complejización de la obra implicaba la intervención de diferentes artistas o creativos y creativas en el proceso de producción o en la fase de promoción (Laddaga, 2006). Los y las artistas visuales que participaron en esta investigación indicaron que, si bien no es frecuente que produzcan en equipos, el establecer redes colaborativas con otros creadores y creadoras ha facilitado la inserción de sus obras en el mercado cultural, así como la captación de un público más amplio, dado que la sinergia se desarrolla como una 
promoción recíproca a través de los contactos que cada agente cultural posee. En el caso de las y los participantes del sub-sector audiovisual, la colaboración, tanto en la fase de producción como en la de la difusión, es más una necesidad que una elección, debido al trabajo creativo y técnico implicado en todo proyecto audiovisual. En el sub-sector del diseño gráfico, según señalaron los y las participantes, la colaboración depende de si el proyecto requiere una mayor planificación o si el diseñador o la diseñadora no cuentan con los recursos o conocimientos técnicos para la realización de un trabajo específico.

\section{Segmentación de mercados y audiencias}

El concepto de segmentación se refiere a la diferenciación de mercados, públicos o consumidores según los rasgos comunes que identifican sus respectivas demandas por algún producto o servicio en particular (Colbert y Cuadrado, 2015). Los factores que influyen en la definición de segmentos, según el marketing cultural, son la dicotomía comprador/no comprador, la frecuencia o la tasa de consumo, el grado de lealtad al producto o a la marca, nivel de satisfacción del consumidor y la preferencia de marca o producto (pp. 123-125). Una vez definido el segmento de mercado, existen dos técnicas de segmentación que Colbert y Cuadrado sugieren emplear: la "a priori" y la de "análisis clúster". La primera consiste en elaborar una hipótesis respecto a las preferencias de los consumidores y comprobarla o refutarla en el mismo proceso de difusión y promoción (p. 132). Por otra parte, el "análisis clúster" no requiere una hipótesis de punto de partida, pero sí un estudio en profundidad del mercado a través del análisis de bases de datos disponibles y, posterior a dicho estudio, se realiza la promoción del producto o servicio cultural (p. 133).

La mayoría de los y las participantes de la investigación afirmaron no conocer metodologías para segmentar sus audiencias o públicos potenciales; no obstante, realizan dicha segmentación de manera intuitiva o con arreglo a criterios que ellos y ellas mismas plantean según sus respectivas experiencias. En el caso de las y los artistas visuales, la diferenciación de públicos la realizan generalmente con base en el poder adquisitivo de sus compradores potenciales, aunque están mayormente interesados en que sus actividades artísticas lleguen a todo público posible. Por su parte, los y las participantes involucrados en el sub-sector audiovisual definen su segmento de mercado de acuerdo a la edad y al grado de estudios de los espectadores y espectadoras potenciales. En lo que respecta a las diseñadoras y los diseñadores gráficos, sí conocen algunos principios mercadológicos básicos, lo que les permite planificar más detalladamente el segmento de mercado al que se dirigirán. Cabe señalar que la totalidad de participantes de este estudio señalaron no contar con las herramientas o con el tiempo para llevar a cabo una investigación de mercados que posibilite una mejor difusión de sus actividades culturales. 


\section{Canales de difusión y promoción}

No existe una definición unívoca del término "canal", dado que es un concepto empleado en diferentes disciplinas y ciencias, tales como la lingüística, la teoría de la comunicación, la informática, etcétera. No obstante, el sentido que se le ha otorgado en esta investigación es el de todo sistema de transmisión de información que posibilita la diseminación de mensajes de un tipo particular de lenguaje mediante un soporte específico (McLuhan, 1996; Thompson, 1998; Briggs y Burke, 2002). En el ámbito de las artes y la cultura, los canales de difusión podrían dividirse en tradicionales u oficiales (Poli, 1976) y digitales. Los primeros se refieren a los medios impresos, así como a espacios físicos y eventos tales como galerías, museos, festivales, certámenes y exposiciones (37); los segundos incluyen redes sociales, mercados virtuales y demás páginas web a través de las cuales los y las agentes culturales promocionan sus actividades.

\section{Canales tradicionales $\mathrm{u}$ oficiales}

Los y las artistas visuales participantes en este estudio señalaron que la presencia en museos, galerías, festivales, concursos y demás espacios y eventos institucionales posibilita una mejor difusión de sus actividades en el mismo campo de producción cultural, es decir, los canales oficiales o tradicionales garantizan, de acuerdo a las y los participantes, el reconocimiento de otros artistas, de críticos, investigadores y demás agentes culturales, lo que conlleva a la validación del o de la artista por sus pares y demás especialistas involucrados en el ámbito cultural. La situación es semejante para los y las participantes que se desempeñan en el subsector audiovisual, quienes aseguran optar por certámenes y festivales cinematográficos organizados por universidades, empresas o instituciones públicas con el objetivo de ser reconocidos por los expertos y expertas del área. Por su parte, las diseñadoras y los diseñadores gráficos no recurren de manera frecuente a los canales tradicionales de difusión y promoción cultural, dado que la última palabra respecto al reconocimiento o validación de sus actividades depende más de la clientela que de los y las especialistas.

\section{Canales digitales}

Si bien son los canales oficiales o tradicionales los que validan las actividades de los y las agentes culturales, como indicaron los y las participantes que laboran en los sub-sectores de las artes visuales y de la producción audiovisual, también comentaron que no siempre es fácil obtener acceso a estos canales, razón por la cual deben optar por los canales digitales que, 
aunque no aseguran el reconocimiento por parte de otros y otras agentes culturales, son más accesibles, principalmente para las creadoras y creadores emergentes. El sesgo que conviene subrayar aquí consiste en que, en el caso de los y las artistas visuales, quienes cuentan con una mayor trayectoria son quienes, a su vez, prefieren los canales tradicionales para difundir y promocionar su trabajo, mientras que los y las artistas nóveles recurren a redes sociales tales como Facebook, Instagram y Twitter, principalmente porque los canales oficiales se centran en artistas reconocidos.

En lo que respecta al ámbito audiovisual, los y las participantes de este estudio señalaron que el canal digital más empleado y efectivo para promocionar su trabajo es Youtube, seguido de Vimeo. Agregaron que recurren a estas plataformas digitales principalmente porque las muestras, certámenes y demás eventos institucionales generalmente tienen una difusión limitada sólo para comunidades universitarias, conocedores y especialistas directamente implicados en el sub-sector audiovisual. En el caso de las diseñadoras y diseñadores gráficos, además de las páginas web ya mencionadas, emplean también DeviantArt y Amino, comunidades virtuales en las que los usuarios y usuarias comparten entre sí sus trabajos. No obstante, el uso que hacen de estos sitios electrónicos es principalmente recreativo o a modo de promoción no formal, mientras que, para fines profesionales, "suben" y comparten con clientes potenciales sus portfolios digitalizados en diferentes páginas de alojamiento de archivos en la "nube", tales como Dropbox, Drive, OneDrive, Mega, etcétera.

\section{Conclusiones}

A través de los resultados que se describieron anteriormente, es posible ensayar una explicación de las razones y motivos que influyen en la elección de los y las agentes culturales respecto a los canales de difusión y promoción que emplean para dar a conocer sus actividades. Los factores que intervienen en la preferencia por un canal en particular son los espacios institucionales y las elaboraciones ideológicas de segundo orden; las universidades, los museos y las galerías son los espacios institucionales de mayor influencia para difundir y promocionar las actividades de los y las agentes culturales, principalmente porque representan modelos de autonomía del arte y de la cultura (Stallabrass, 2004). Es decir, a través de estos espacios es que se reivindica la independencia de la producción cultural respecto de las demás esferas de la actividad humana, así como la finalidad de la cultura como una serie de prácticas encaminadas al desarrollo integral del ser humano, visión legada por el proyecto ilustrado (Bauman, 2002).

Las elaboraciones ideológicas tales como reseñas, comentarios, artículos, notas y demás textos, son construcciones discursivas que acreditan o desacreditan determinadas prácticas 
como pertenecientes a un ámbito específico de la praxis humana (Richard, 2007). En Nuevo León, la crítica cultural y artística no tiene un alcance masivo, y generalmente está limitada al mismo campo de producción cultural, siendo las comunidades universitarias, así como los y las agentes culturales, los principales destinatarios de este tipo de publicaciones. Pese a que la presencia en espacios institucionales y los comentarios, críticas, reseñas, etcétera, son de los factores principales que garantizan una mejor difusión y promoción de las actividades de los y las agentes culturales, el acceso a los espacios institucionales generalmente se reserva para creadores y creadoras en consolidación o consolidados, mientras que las y los agentes culturales emergentes tienen a su disposición muy pocos espacios para dar a conocer su trabajo. En lo que respecta a las elaboraciones ideológicas secundarias, además de la universidad y de algunos medios institucionales públicos, existe poca o nula cobertura en la forma de textos o escritos que se refieran al trabajo de las y los agentes culturales en el estado. En el sub-sector del diseño gráfico, los comentarios que validan el trabajo de las diseñadoras y de los diseñadores son aquellos realizados por la clientela misma, y no se refieren a cuestiones de índole estética, sino a criterios de tipo profesional, tales como cumplimiento de la labor asignada, condiciones de entrega, precios, etcétera.

Además de los factores ya mencionados, otro aspecto importante a considerar en la elección de los canales de difusión y promoción cultural es el de las estrategias más recurrentes para la inserción del bien o servicio cultural o para la captación de mayores audiencias. En este estudio, las tres tácticas más frecuentemente empleadas por los y las agentes culturales participantes son las de la personalización, la colaboración y la segmentación, siendo la primera la estrategia preferida por los y las participantes que laboran en el sub-sector del diseño gráfico, principalmente porque atienden a la demanda de clientes potenciales. En el caso de los y las artistas visuales, así como de los creadores y creadoras audiovisuales, prefieren establecer redes colaborativas con el propósito de promocionar sus actividades de manera recíproca y así ampliar la recepción de las audiencias potenciales. Por otra parte, la estrategia de segmentación es aplicada en menor medida, aunque sin una metodología explícita, en base a criterios intuitivos. Cabe agregar que los agentes culturales de los subsectores artístico-visual y audiovisual tienen por objetivo un público generalizado, por lo que la diferenciación de segmentos no es usual.

Dado que el acceso a espacios institucionales es restringido y la cobertura de los medios en torno al ámbito cultural es reducida, los y las agentes culturales deben buscar otras formas para dar a conocer sus actividades a un público más amplio. Los canales tradicionales $u$ oficiales generalmente son restrictivos, entre otras razones porque son financiados por presupuesto público o porque requieren obtener ganancias para recuperar la inversión realizada en un evento cultural o en la promoción de artistas o videastas. Además, dada la insuficiencia de empleos en el ámbito de la cultura, así como la escasez de apoyos públicos o 
patrocinios a la creación cultural (Maanen, 2009), los y las agentes culturales se ven en la necesidad de recurrir a canales digitales para promocionar sus actividades y así lograr captar incluso audiencias ajenas al campo de producción cultural. Si bien los y las participantes reconocen que son los canales tradicionales $u$ oficiales los que validan el trabajo de una o un agente cultural, admiten también que, en términos de difusión y promoción, los canales digitales proveen una más amplia cobertura.

Más que una preferencia, el empleo de canales digitales como vías de difusión y promoción cultural es, en cierta medida, una necesidad. Son diversas las circunstancias que propician la elección de los entornos electrónicos por parte de los y las agentes culturales: la desmaterialización de la obra, que implica el hecho de que la digitalización sea una opción más económica para los creadores y creadoras, la deslocalización o el que a través de Internet sea posible acortar distancias geográficas antes insalvables, el hecho de que la producción cultural encuentra en los entornos digitales una ecosistema flexible acorde a los ritmos del trabajo creativo (Rama, 1999). Todo ello implica que el campo de la producción cultural experimenta una transición de una estructura organizativa a otra basada en las capacidades estéticas, colaborativas y conectivas del Internet (Goriunova, 2012). Aun así, y a partir de este estudio que se circunscribe a un espacio geográfico, político y cultural específico, Nuevo León, estado norteño de México, dicha transición es apenas un proceso que comienza a gestarse en un momento clave en el que las creadoras y los creadores culturales emergentes proponen nuevos modos de producción, a la vez que buscan establecer nuevos canales de difusión, todo ello con el propósito de generar nuevas formas de interacción con un público local.

\section{Agradecimientos}

La presente investigación ha sido realizada gracias al Apoyo de Fomento a la Generación y Aplicación Innovadora del Conocimiento y al Fortalecimiento de Cuerpos Académicos, ambos patrocinios pertenecientes al Programa para el Desarrollo Profesional Docente, para el Tipo Superior (PRODEP), y por el Programa de Apoyo a la Investigación Científica y Tecnológica (PAICyT) de la Universidad Autónoma de Nuevo León. El autor desea agradecer también a los entrevistados que accedieron a participar en este estudio.

\section{Bibliografía}

Alexander, J. C. (2000). Sociología cultural. Formas de clasificación en las sociedades complejas. 
Barcelona: Anthropos.

Álvarez-Gayou Jurgenson, J. L. (2012). Cómo hacer investigación cualitativa. Fundamentos y metodología. México: Paidós.

Amores, J. E. (2007). Monterrey: una cultura propia. En Ortega Ridaura, I. (coord.). Nuevo León en el siglo XX. Del Segundo auge industrial a la crisis de 1982 (pp. 73-107). Monterrey: Fondo Editorial de Nuevo León.

Bauman, Z. (2002). La cultura como praxis. Barcelona: Paidós.

Becker, H. S. (2008). Los mundos del arte. Sociología del trabajo artístico. Buenos Aires: Universidad Nacional de Quilmes.

Bernal, C. A. (2010). Metodología de la investigación. Bogotá: Pearson.

Bourriaud, N. (2008). Estética relacional. Buenos Aires: Adriana Hidalgo Editora.

Briggs, A. y Burke, P. (2002). De Gutenberg a Internet. Una historia social de los medios de comunicación. Madrid: Taurus.

Castells, M. (2001). La galaxia Internet. Barcelona: Plaza \& Janés.

Colbert, F. y Cuadrado, M. (2015). Marketing de las artes y la cultura. Barcelona: Ariel.

De Certeau, M. (1999). La cultura en plural. Buenos Aires: Nueva Visión.

Faber, S. (2009). Ideología. En Szurmuk, M. y Mckee Irwin, R. (coords.). Diccionario de estudios culturales latinoamericanos. México: Siglo XXI.

García Canclini, N. (2014). La producción simbólica. Teoría y método en sociología del arte. México: Siglo XXI.

Goffman, E. (1974). Frame Analysis. An Essay on the Organization of Experience. New York: Harper Colophon Books.

Goriunova, O. (2012). Art Platforms and Cultural Production on the Internet. New York: Routledge.

Howkins, J. (2013). The Creative Economy. How People Make Money from Ideas. London: Penguin Books.

Laddaga, R. (2006). Estética de la emergencia. La formación de otra cultura de las artes. Buenos Aires: Adriana Hidalgo Editora.

Maanen, H. (2009). How to Study Art Worlds. On the Societal Functioning of Aesthetic Values. Amsterdam: Amsterdam University Press.

McLuhan, M. (1996). Comprender los medios de comunicación. Las extensiones del ser humano. Barcelona: Paidós.

Nivón Bolán, E. y Villalobos Audifred, H. (2006). Perfil metropolitano del consumo cultural. Los casos de Guadalajara, Monterrey y el Distrito Federal. En Arizpe, L. (coord.). Retos Culturales de México frente a la globalización (pp. 535-559). México: Miguel Ángel Porrúa.

Poli, F. (1976). Producción artística y mercado. Barcelona: Gustavo Gili. 
PNUD (2014). Informe sobre la economía creativa. UNESCO. Recuperado de http://www.unesco.org/culture/pdf/creative-economy-report-2013-es.pdf

Rama, C. (1999). El capital cultural en la era de la globalización digital. Montevideo: Arca.

Richard, N. (2007). Fracturas de la memoria. Arte y pensamiento crítico. Buenos Aires: Siglo $\mathrm{XXI}$.

Rubio Elosúa, E. (2000). De promotores, instituciones y políticas culturales. En Moyssén, X. (ed.). Artes Plásticas de Nuevo León. 100 años de historia, siglo XX (pp. 155-196). Monterrey: Museo de Monterrey.

Stallabrass, J. (2004). Art Incorporated. The Story of Contemporary Art. New York: Oxford University Press.

Thompson, J. B. (1998). Los media y la modernidad. Una teoría de los medios de comunicación. Barcelona: Paidós.

Valles, M. (1999). Técnicas cualitativas de investigación social. Reflexión metodológica y práctica social. Madrid: Síntesis.

Williams, R. (1981). Cultura. Sociología del arte y de la comunicación. Barcelona: Paidós. 